\title{
Highly sensitive detection of protein biomarkers via nuclear magnetic resonance biosensor with magnetically engineered nanoferrite particles
}

This article was published in the following Dove Press journal:

International Journal of Nanomedicine

21 October 2016

Number of times this article has been viewed

\author{
Minhong Jeun' \\ Sungwook Park ${ }^{1,2}$ \\ Hakho Lee ${ }^{3}$ \\ Kwan Hyi Lee ${ }^{1,2}$ \\ 'Center for Biomaterials, Biomedical \\ Research Institute, Korea Institute \\ of Science and Technology, Seoul, \\ ${ }^{2}$ Department of Biomedical \\ Engineering, Korea University of \\ Science and Technology, Daejeon, \\ Republic of Korea; ${ }^{3}$ Center for \\ Systems Biology, Massachusetts \\ General Hospital, Harvard Medical \\ School, Boston, MA, USA
}

\begin{abstract}
Magnetic-based biosensors are attractive for on-site detection of biomarkers due to the low magnetic susceptibility of biological samples. Here, we report a highly sensitive magneticbased biosensing system that is composed of a miniaturized nuclear magnetic resonance (NMR) device and magnetically engineered nanoferrite particles (NFPs). The sensing performance, also identified as the transverse relaxation $\left(R_{2}\right)$ rate, of the NMR device is directly related to the magnetic properties of the NFPs. Therefore, we developed magnetically engineered NFPs (MnMg-NFP) and used them as NMR agents to exhibit a significantly improved $R_{2}$ rate. The magnetization of the MnMg-NFPs was increased by controlling the Mn and Mg cation concentration and distribution during the synthesis process. This modification of the $\mathrm{Mn}$ and $\mathrm{Mg}$ cation directly contributed to improving the $R_{2}$ rate. The miniaturized NMR system, combined with the magnetically engineered MnMg-NFPs, successfully detected a small amount of infectious influenza A H1N1 nucleoprotein with high sensitivity and stability.
\end{abstract}

Keywords: biosensor, NMR, nanoferrite particles, transverse relaxation, magnetization, onsite detection

\section{Introduction}

On-site detection for rapid initial examination regarding possible virus infection is critical in preventing serious contagions. Accordingly, there has been an increase in the development of sensitive biosensor systems that include electrical, optical, and magnetic-based mechanisms. ${ }^{1-6}$ In particular, the magnetic-based sensing system is an attractive method for biosensor applications. This is possible because intrinsically biological media have low magnetic susceptibility. Consequently, the magnetic sandwich assay can achieve a high detection signal with low interferences even in a complex biological background. ${ }^{7}$

Nuclear magnetic resonance (NMR) is a useful magnetic-based sensing technology that enables rapid, stable, and highly sensitive biomarker detection by utilizing magnetic resonance technology to detect biomarkers labeled with magnetic particles. ${ }^{8,9}$ The transverse relaxation $\left(R_{2}\right)$ rate of the water protons in the vicinity of the magnetic particles and the target biomarker is modified upon reacting with the magnetic particles, and this change can be detected by the NMR. The biomarkers labeled with magnetic particles exhibit a faster decay rate (larger $R_{2}$ ) of NMR signals than that of non-magnetic particlelabeled biomarkers. ${ }^{10-12}$ The NMR-based detection technology requires minimal sample purification steps and consequently reduces sample loss. In addition, because the NMR signal is generated from the whole volume, the binding kinetics for the NMR signal 
is much quicker than that of surface reaction-based sensors. This allows the NMR assay the advantage of obtaining a rapid sensing signal at a faster pace. Naturally, the NMR signal is directly relevant to the magnetic particles. Because magnetic particles with a higher $R_{2}$ rate can improve the detection sensitivity in NMR-based sensing, developing a magnetic NMR agent that exhibits a higher $R_{2}$ rate is imperative in achieving maximal sensitivity in the NMR sensing.

In this study, we developed a high-performance magnetic particle to improve the NMR sensitivity and then demonstrated its feasibility as an NMR agent by conducting a detection test for the nucleoproteins of the influenza A H1N1 virus. In order to achieve this goal, we magnetically engineered Fe-nanoferrite particles (Fe-NFPs) to enhance the $R_{2}$ rate. The Fe ions in tetrahedral A $\left(\mathrm{T}_{\mathrm{A}}\right)$ sites or octahedral $\mathrm{B}\left(\mathrm{O}_{\mathrm{B}}\right)$ sites were substituted with $\mathrm{Mn}$ ions (Mn-NFPs) and Mn/Mg ions (MnMg-NFPs) for increased magnetization; the $R_{2}$ rate of the NFPs is proportional to the magnetization $\left(R_{2} \approx d^{2} M^{2} ; d\right.$ represents the diameter of the nanoparticle). ${ }^{13}$

\section{Materials and methods}

\section{Synthesis of nanoferrites and surface modification}

All the NFPs were synthesized using a high temperature thermal decomposition method. ${ }^{14,15}$ The metal precursors (Fe (III) acetylacetonate $\left[\left(\mathrm{C}_{5} \mathrm{H}_{8} \mathrm{O}_{2}\right)_{3} \mathrm{Fe}\right](>99.9 \%), \mathrm{Mn}$ (II) acetate tetrahydrate $\left[\left(\mathrm{CH}_{3} \mathrm{COO}\right)_{2} \mathrm{Mn}_{4} \mathrm{H}_{2} \mathrm{O}\right](99.99 \%)$ and $\mathrm{Mg}$ acetate tetrahydrate $\left.\left[\left(\mathrm{C}_{2} \mathrm{H}_{3} \mathrm{O}_{2}\right)_{2} \mathrm{Mg}_{4} \mathrm{H}_{2} \mathrm{O}\right](99.999 \%)\right)$ and other materials such as the surfactants (oleic acid $\left[\mathrm{C}_{18} \mathrm{H}_{34} \mathrm{O}_{2}\right]$ $(90 \%)$, oleylamine $\left.\left[\mathrm{C}_{18} \mathrm{H}_{37} \mathrm{~N}\right](70 \%)\right)$, reducing agents $(1,2-$ hexadecanediol $\left.\left[\mathrm{CH}_{3}\left(\mathrm{CH}_{2}\right)_{13} \mathrm{CH}(\mathrm{OH}) \mathrm{CH}_{2} \mathrm{OH}\right](90 \%)\right)$, and solvent (benzyl ether $\left[\mathrm{C}_{14} \mathrm{H}_{14} \mathrm{O}\right](99 \%)$ ) were mixed and heated up to $296^{\circ} \mathrm{C} .{ }^{11}$ The $\mathrm{Mn}$ and $\mathrm{Mg}$ cation concentration and distribution in Mn-NFPs and MnMg-NFPs were chemically controlled by adjusting the amount of the Mn and $\mathrm{Mg}$ precursors and the amount of the reducing agent during the synthesis process. Then, all the synthesized NFPs were rendered water soluble by coating the surface of particles with polyethylene glycol (PEG).

Water-soluble NFPs were obtained by forming a lipid layer (PEGylated lipid) with 1-myristoyl-2-hydroxy-snglycero-3-phosphocholine/1,2-dipalmitoyl-sn-glycero-3phosphoethanolamine-N-(lauroyl)-polyethylene glycol 2000 (MHPC/DSPE-PEG2K). The NFPs were conjugated with influenza A H1N1 nucleoprotein antibodies. The reaction of the primary amines on the antibody with PEG-coated
NFPs (NFPs@PEG) was catalyzed by 1-ethyl-3-[3-dimethylaminopropyl]carbodiimide hydrochloride and sulfo- $N$ hydroxysuccinimide. More detailed protocols regarding the surface modification of NFPs and antibody conjugation with NFPs are indicated in our previous reports. ${ }^{16-18}$

\section{Characterization of nanoferrites}

The size and size distribution of the synthesized NFPs were measured by transmission electron microscopy. The hydrodynamic diameter $\left(d_{\mathrm{H}}\right)$ and polydispersity index (PDI) of the NFPs@PEG were measured using a dynamic light scattering system. The magnetic hysteresis characteristics, including the major and minor hysteresis loops for both the non-coated (powder state) and PEG-coated (fluid state) NFPs, were investigated using a vibrating sample magnetometer. The $R_{2}$ rate was measured by employing a miniaturized NMR system. ${ }^{19}$ The NMR system consists of three components, microcoils, on-board NMR electronics, and a small permanent magnet. The permanent magnet can be used to generate polarizing magnetic field, $B_{0}=0.1-0.5 \mathrm{~T}$. The concentrations of NFPs for the measurement of $R_{2}$ rate were varied from $10^{-2}$ to $10^{-11} \mathrm{~g} / \mathrm{mL}$.

\section{Relaxation measurement}

The biosensing performances, including sensitivity and limit-of-detection (LOD) of the NFPs, were evaluated by detecting the nucleoproteins of influenza A H1N1 virus using the miniaturized NMR system combined with the magnetic sandwich method. The H1N1 nucleoprotein and antibody to the H1N1 nucleoproteins were purchased from Sino Biological Inc. A total of $15 \mu \mathrm{L}$ of the H1N1 nucleoprotein mixture solution was first mixed with polystyrene beads (diameter: $3 \mu \mathrm{m}$ ) conjugated with the capture antibody and incubated for $30 \mathrm{~min}$ at room temperature. Uncaptured H1N1 nucleoproteins were removed by washing the beads with the wash buffer. The NFPs conjugated with detection antibody were then added, and the mixture was incubated again for 20 min. The unbound NFPs were removed by washing and filtering the mixture with the wash buffer, and finally, $15 \mu \mathrm{L}$ of phosphate-buffered saline (PBS) was added to the mixture for NMR measurement (Scheme 1). We carried out all $R_{2}$ measurements at the polarizing magnetic field of $B_{0}=0.5 \mathrm{~T}$. All the measurements were done in triplicate at room temperature.

\section{Results and discussion}

As the coating and dispersion status of the particles inside the fluid affects the magnetic properties, we investigated the PEG 


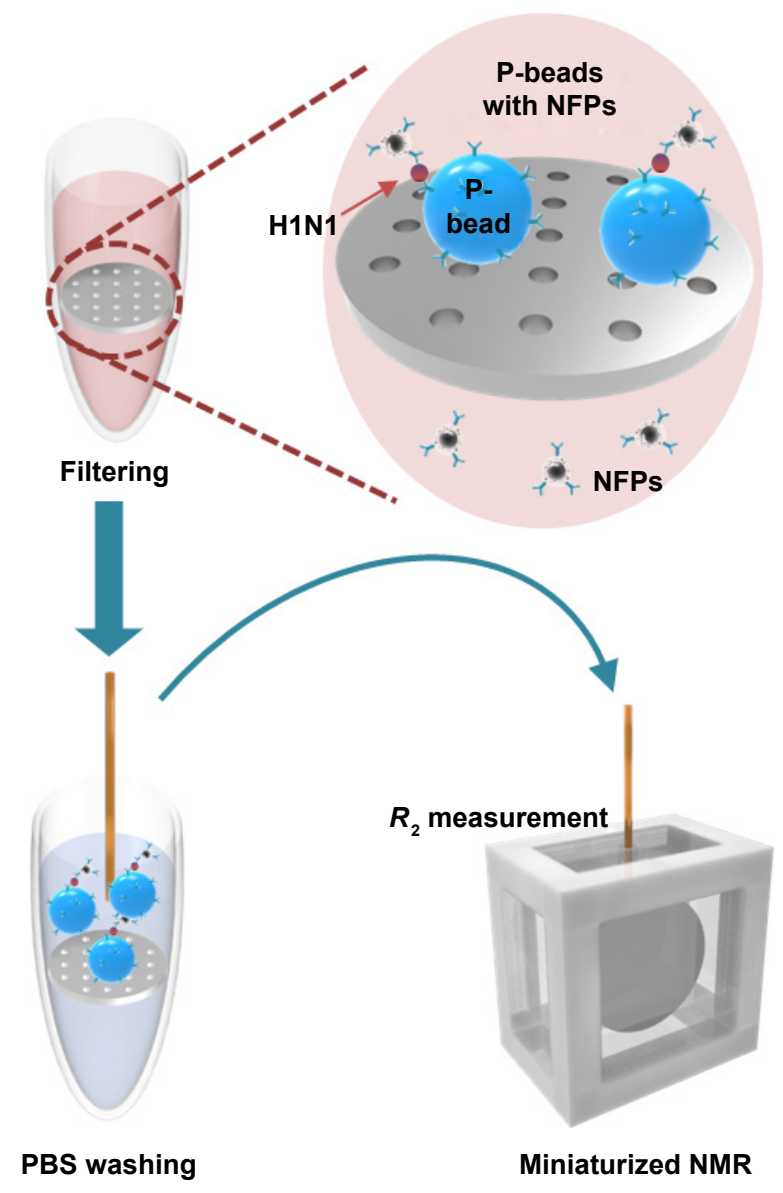

Scheme I Schematic illustration to show the detecting procedure for the influenza A HINI nucleoprotein using the miniaturized NMR system. Abbreviations: NMR, nuclear magnetic resonance; NFP, nanoferrite particle; PBS, phosphate-buffered saline.

coating status for all the PEG-coated NFPs (NFPs@PEG). All the NFPs@PEG had a similar size, size distribution, and PDI. As can be seen in Figure 1A-C, all the NFPs@PEG dispersed in deionized (DI) water had similar small-sized nanoferrite cores (diameter: 7-10 nm) and uniform $d_{\mathrm{H}}$ of $35.3-37.5 \mathrm{~nm}$ with a narrow size distribution of $\sim 10.16 \%$ and low PDI values in the range of 1.15-1.28, indicating that the NFPs were uniformly coated with the PEG and that the NFPs@PEG were well dispersed in DI water with minimal aggregation.

Figure 2 shows the direct current (DC) magnetic hysteresis loops of the A powder state and the B fluid state of the NFPs measured at room temperature. The synthesized NFPs did not show any DC minor hysteresis at the sweeping field of $\pm 140 \mathrm{Oe}$, indicating that all the nanoparticles synthesized in this study have a pure superparamagnetic phase (Figure 2A inset). Among all the NFPs, in particular, the $\mathrm{MnMg}$-NFPs showed a higher magnetization than that of the
Mn-NFPs and Fe-NFPs. This result implies that the MnMgNFPs are magnetically softer and have a lower magnetic anisotropy (or a higher DC magnetic susceptibility, $\chi_{\mathrm{m}}$ ) than that of the Mn-NFPs and Fe-NFPs. Considering that the preferential site of $\mathrm{Mn}^{2+}$ cations is the $\mathrm{O}_{\mathrm{B}}$ site of Fe-NFPs, the improved magnetization of Mn-NFPs is physically thought to be due to the possible substitution of $\mathrm{Fe}^{2+}$ cations with $4 \mu_{\mathrm{B}}$ (Bohr magneton) in the $\mathrm{O}_{\mathrm{B}}$ sites by $\mathrm{Mn}^{2+}$ cations with $5 \mu_{\mathrm{B}}$ during the synthesis process (Figure 2C).

In the case of the MnMg-NFPs, where the preference site of $\mathrm{Mg}^{2+}$ cations is the $\mathrm{T}_{\mathrm{A}}$ site, the $\mathrm{Mg}^{2+}$ cations $\left(\mu_{\mathrm{B}}=0\right)$ replace the $\mathrm{Fe}^{2+}$ cations in the $\mathrm{T}_{\mathrm{A}}$ site of Mn-NFPs, and as a result, the magnetic moment of the MnMg-NFPs increases $\left(6.2 \mu_{B}\right.$, total net $\mu_{B}=\mu_{B}\left[O_{B}\right.$ site $]-\mu_{B}\left[T_{A}\right.$ site $\left.]\right)$. The fluid state NFPs@PEG (Figure 2B) also retains the superparamagnetic phase and stable DC magnetic hysteresis loops. This is due to the superior PEG coating status, which allows for a more uniform anti-body conjugation to the NFPs@PEG without spontaneous magnetic aggregation. ${ }^{20,21}$

The $R_{2}$ characteristic of each NFPs@PEG was measured using the miniaturized NMR system in a magnetic field $B_{0}$ $(0.5 \mathrm{~T})$ to evaluate the feasibility as an NMR agent for the detection of H1N1 nucleoproteins. In order to measure the $R_{2}$ rate, Fe-NFPs@PEG, Mn-NFPs@PEG, and MnMgNFPs@PEG were dispersed in DI water. The concentration of the NFPs@PEG dispersed in DI water varied from $5 \times 10^{-2} \mathrm{~g} / \mathrm{mL}$ to $5 \times 10^{-10} \mathrm{~g} / \mathrm{mL}$. Figure 3 shows the $R_{2}$ values for the three NFPs@PEGs at various concentrations. The observed $R_{2}$ values increased as the concentration increased, and the MnMg-NFPs@PEG exhibited the highest $R_{2}$ value among the different NFPs@PEGs.

Considering the fact that the $R_{2}$ is proportional to magnetization $\left(R_{2} \approx d^{2} M^{2}\right)$, the highest $R_{2}$ value of the MnMgNFPs@PEG is thought to be due to the highly improved magnetization caused by engineering the cation concentration and distribution in the NFPs. This result demonstrates that the MnMg-NFPs@PEG is high enough to be considered as an NMR agent.

In order to demonstrate the applicability of the engineered NFPs as an NMR agent, we used the MnMg-NFPs@PEG and the Fe-NFPs@PEG to detect H1N1 nucleoproteins in a miniaturized NMR system. Figure 4A shows the miniaturized NMR system and representative NMR signal (T2 time of MnMgNFPs@PEG). Figure 4B exhibits the H1N1 nucleoprotein detection ability $\left(\Delta R_{2}=R_{2, \mathrm{P}-\text { beads }+\mathrm{H} 1 \mathrm{~N} 1+\mathrm{NFPs}}-R_{2, \mathrm{P}-\text { beads }}\right)$ of the MnMg-NFPs@PEG and Fe-NFPs@PEG. Both NFPs@PEGs successfully detected the amount of H1N1 nucleoprotein with 

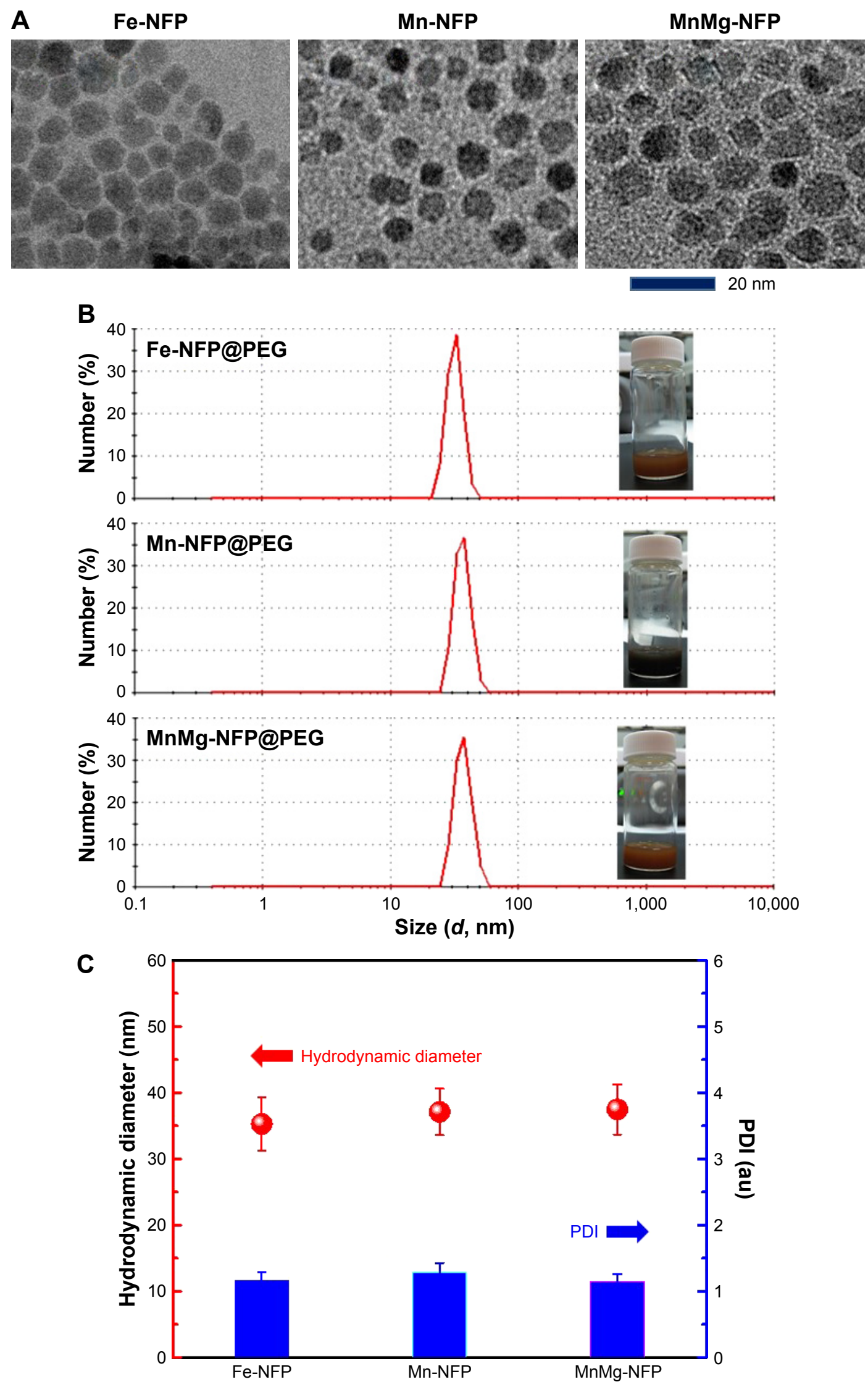

Figure I (A) Sizes of synthesized NFPs measured by TEM, (B) size distribution, and (C) hydrodynamic diameter and PDI of the PEG-coated NFPs characterized by a DLS system.

Abbreviations: NFP, nanoferrite particle; TEM, transmission electron microscopy; PDI, polydispersity index; PEG, polyethylene glycol; DLS, dynamic light scattering. 

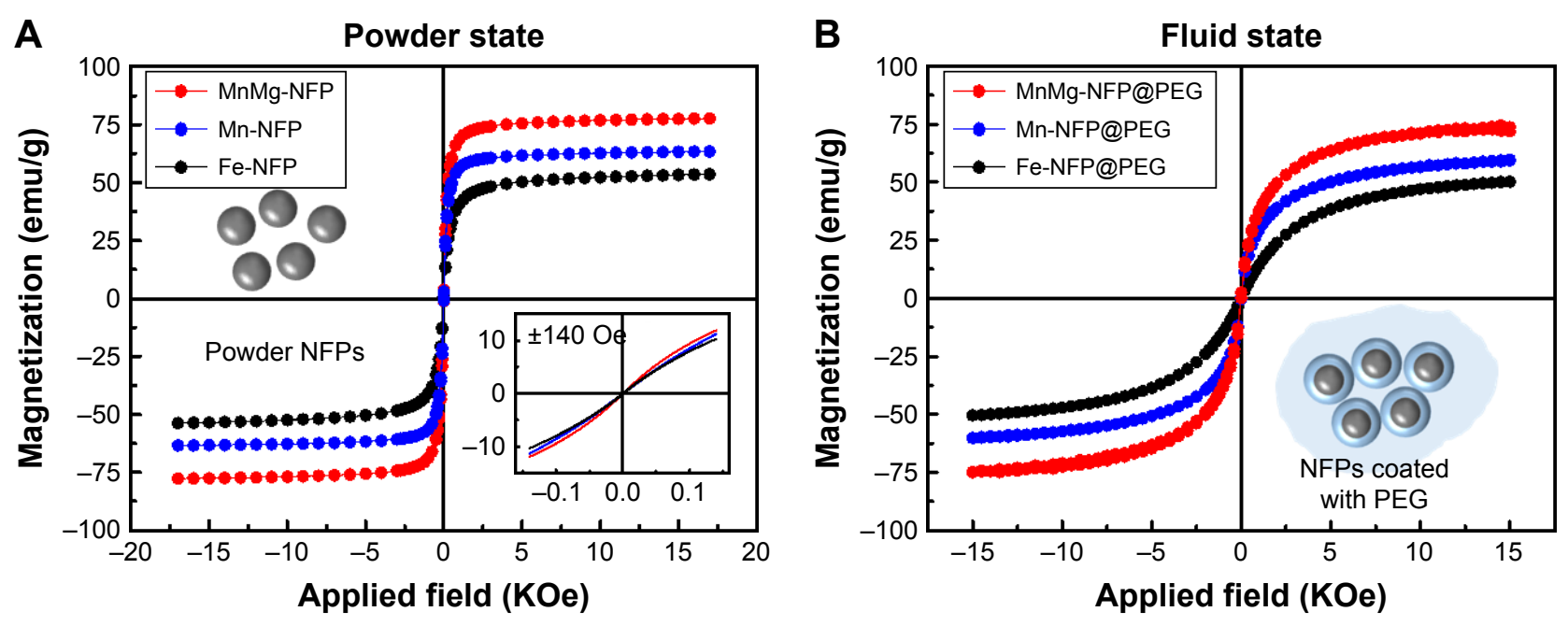

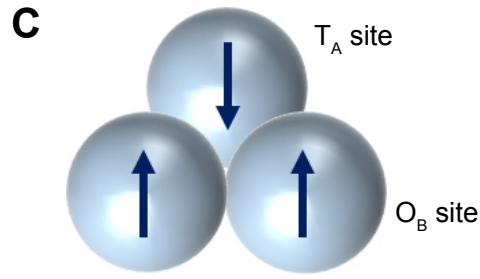

Fe-NFP $4 \mu_{\text {B }}$

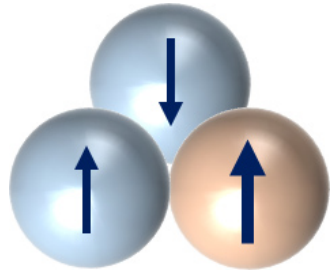

Mn-NFP $5 \mu_{B}$

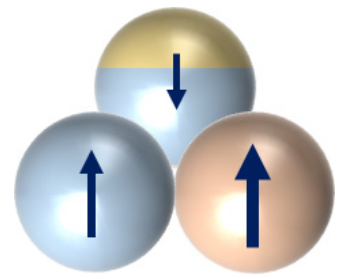

MnMg-NFP $6.2 \mu_{B}$

Figure 2 Major and minor magnetic hysteresis loops of the prepared NFPs measured in the (A) powder state and (B) fluid state. (C) Schematic illustrations to express the magnetic moment of the NFPs depending on the cation concentration and distribution. $\mu_{B}$, Bohr magneton; $T_{A}$, tetrahedral $A$ site; $O_{B}$, octahedral $B$ sites. Abbreviations: NFP, nanoferrite particle; PEG, polyethylene glycol.

a low error range of $<4.5 \%$. The H1N1 nucleoproteins bound to a polystyrene bead can be a cause of the aggregation of NFPs and the corresponding increase in the $R_{2}$ rate.

As can be clearly seen in the result, the MnMg-NFPs@ PEG showed a significantly higher $\Delta R_{2}$, a wider dynamic

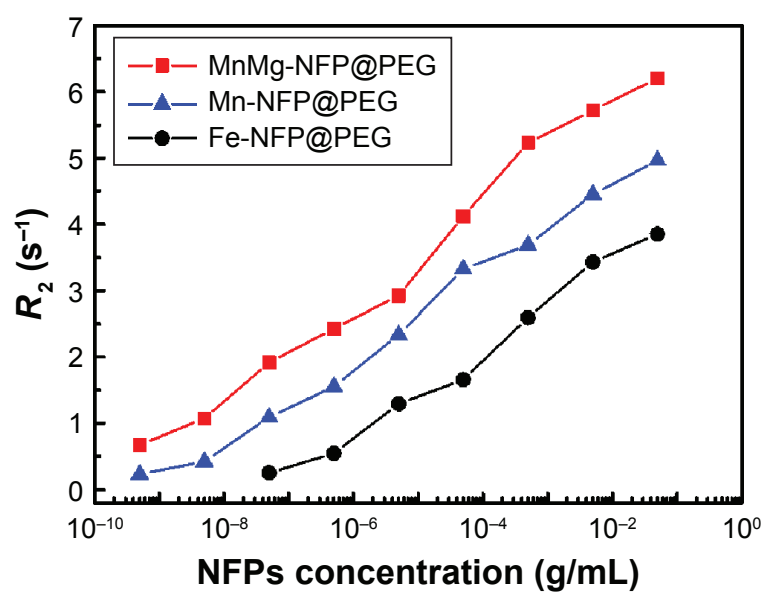

Figure 3 Transverse relaxation $\left(R_{2}\right)$ rate of all the NFPs@PEG depending on the concentration.

Note: The $R_{2}$ rate was measured by the miniaturized NMR system.

Abbreviations: NFP, nanoferrite particle; PEG, polyethylene glycol; NMR, nuclear magnetic resonance. range, and a lower LOD than those of Fe-NFPs@PEG. The MnMg-NFPs@PEG showed a $\Delta R_{2}$ of $0.501 \mathrm{~s}^{-1}(\mathrm{~g} / \mathrm{mL})^{-1}$ with an LOD of $1 \times 10^{-9} \mathrm{~g} / \mathrm{mL}$, while the Fe-NFPs exhibited a $\Delta R_{2}$ of $0.329 \mathrm{~s}^{-1}(\mathrm{~g} / \mathrm{mL})^{-1}$ with an LOD of $1 \times 10^{-7} \mathrm{~g} / \mathrm{mL}$. All the results strongly demonstrate that the magnetically engineered MnMg-NFPs have the potential to be a promising NMR agent for the detection of viral biomarkers with high sensitivity and stability.

\section{Conclusion}

We developed the magnetically engineered MnMg-NFPs for application as an NMR agent to detect infectious viruses. The $\mathrm{Mn}$ and $\mathrm{Mg}$ cation concentration and distribution in the MnMg-NFPs were chemically controlled during the Fe-NFP synthesis to improve the magnetization of spinelstructured NFPs. As a result, the MnMg-NFPs showed the highest magnetization value among the prepared NFPs, as well as a correspondingly improved $R_{2}$ rate. The potential of the MnMg-NFPs as an NMR agent was demonstrated by conducting the $\mathrm{H} 1 \mathrm{~N} 1$ nucleoprotein detection test. The MnMg-NFPs successfully detected a tiny amount of H1N1 nucleoproteins in PBS with a stable and high $R_{2}$ rate (high sensitivity). 

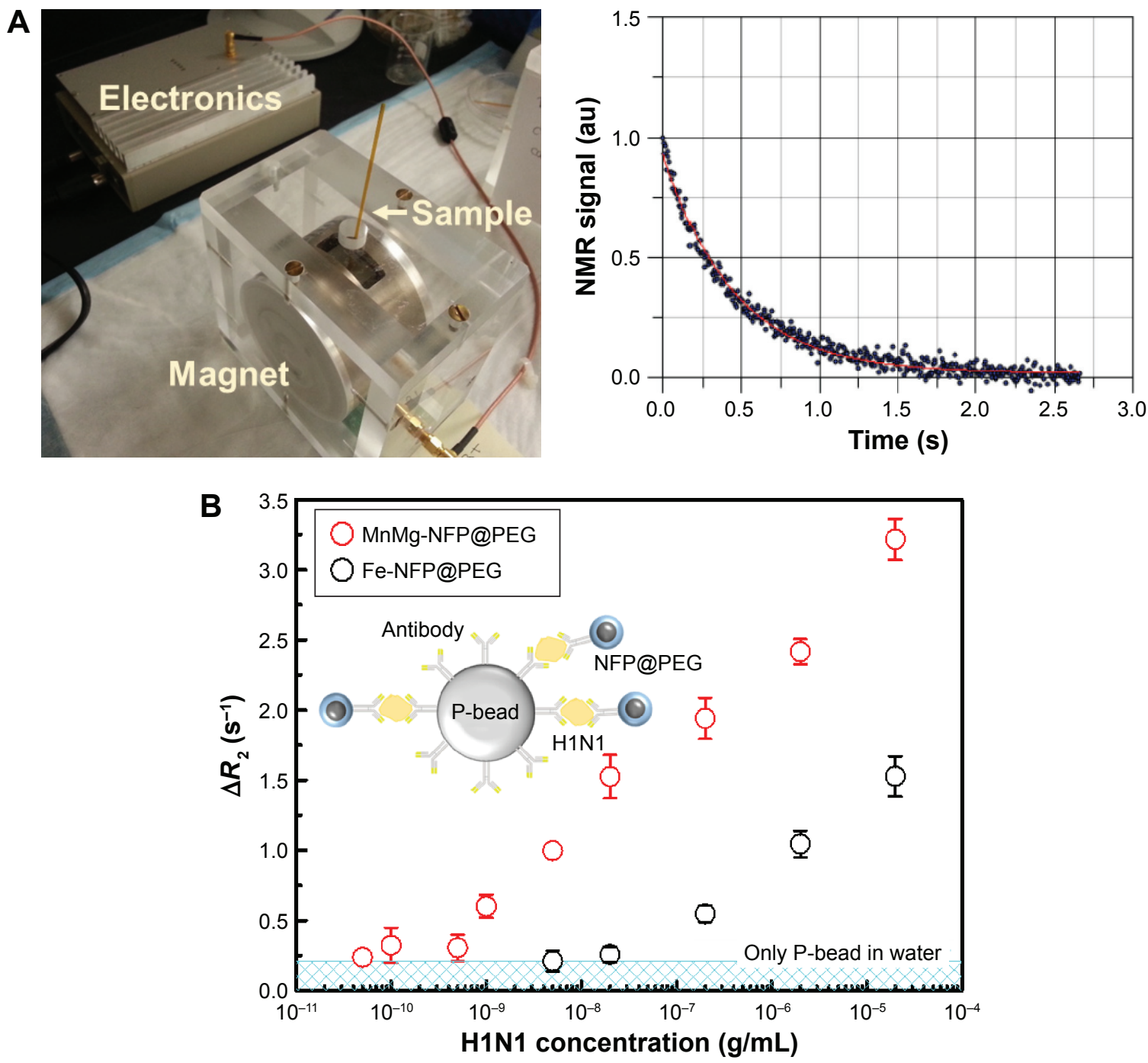

Figure 4 (A) Miniaturized NMR system and representative measured NMR signal (T2 time). (B) Detection results of influenza A HINI nucleoprotein using the miniaturized NMR system with MnMg-NFPs@PEG and Fe-NFPs@PEG. The MnMg-NFPs@PEG and Fe-NFPs@PEG are conjugated with the detection antibodies and used as an NMR agent. The polystyrene bead is conjugated with the capture antibodies.

Abbreviations: NMR, nuclear magnetic resonance; NFP, nanoferrite particle; PEG, polyethylene glycol.

\section{Acknowledgments}

This research was supported by the Basic Science Research Program through the National Research Foundation of Korea (NRF) funded by the Ministry of Education (2013R1A6A3A04063923), the Bio \& Medical Technology Development Program of the NRF funded by the Korean government MSIP (2015M3A9E2029265), and Korea Health Technology R\&D Project through the Korea Health Industry Development Institute funded by the Ministry of Health and Welfare, Republic of Korea (HI15C-3078-020015).

\section{Disclosure}

The authors report no conflicts of interest in this work.

\section{References}

1. Allegranzi B, Nejad SB, Combescure C, et al. Burden of endemic health-care-associated infection in developing countries: systematic review and meta-analysis. Lancet. 2011;377(9761):228-241.

2. Nguyen TT, Bea SO, Kim DM, et al. A regenerative label-free fiber optic sensor using surface plasmon resonance for clinical diagnosis of fibrinogen. Int J Nanomedicine. 2015;10(Spec Iss):155-163.

3. Klompas M, Yokoe DS. Automated surveillance of health careassociated infections. Clin Infect Dis. 2009;48(9):1268-1275.

4. Apetrei IM, Apetrei C. Voltammetric determination of melatonin using a graphene-based sensor in pharmaceutical products. Int J Nanomedicine. 2016;11:1859-1866.

5. Polin RA, Denson S, Brady MT, Newborn CF, Dis CI. Epidemiology and diagnosis of health care-associated infections in the NICU. Pediatrics. 2012;129(4):1104-1109.

6. Mers SS, Kumar ET, Ganesh V. Gold nanoparticles-immobilized, hierarchically ordered, porous $\mathrm{TiO}_{2}$ nanotubes for biosensing of glutathione. Int J Nanomedicine. 2015;10(suppl 1):171-182. 
7. Min C, Shao HL, Liong M, Yoon TJ, Weissleder R, Lee H. Mechanism of magnetic relaxation switching sensing. ACS Nano. 2012;6(8): 6821-6828.

8. Lee H, Yoon TJ, Figueiredo JL, Swirski FK, Weissleder R. Rapid detection and profiling of cancer cells in fine-needle aspirates. Proc Natl Acad Sci U S A. 2009;106(30):12459-12464.

9. Lee H, Yoon TJ, Weissleder R. Ultrasensitive detection of bacteria using core-shell nanoparticles and an NMR-filter system. Angew Chem Int Edit. 2009;48(31):5657-5660.

10. Gillis P, Koenig SH. Transverse relaxation of solvent protons induced by magnetized spheres - application to ferritin, erythrocytes, and magnetite. Magn Reson Med. 1987;5(4):323-345.

11. Koenig SH, Kellar KE. Theory of $1 / \mathrm{T}_{1}$ and $1 / \mathrm{T}_{2}$ NMRD profiles of solutions of magnetic nanoparticles. Magn Reson Med. 1995;34(2):227-233.

12. Yang HC, Liu CW, Liao SH, et al. Temperature and concentrationdependent relaxation of ferrofluids characterized with a high-Tc SQUID-based nuclear magnetic resonance spectrometer. Appl Phys Lett. 2012;100(20):202405-202408.

13. Gillis P, Moiny F, Brooks RA. On $\mathrm{T}_{2}$-shortening by strongly magnetized spheres: a partial refocusing model. Magn Reson Med. 2002;47(2): 257-263.

14. Jeun M, Park S, Jang GH, Lee KH. Tailoring $\mathrm{Mg}_{\mathrm{x}} \mathrm{Mn}_{1-\mathrm{x}} \mathrm{Fe}_{2} \mathrm{O}_{4}$ superparamagnetic nanoferrites for magnetic fluid hyperthermia applications. ACS Appl Mater Inter. 2014;6(19):16487-16492.
15. Jeun M, Moon SJ, Kobayashi H, et al. Effects of Mn concentration on the ac magnetically induced heating characteristics of superparamagnetic $\mathrm{Mn} \mathrm{Zn}_{1-\mathrm{x}} \mathrm{Fe}_{2} \mathrm{O}_{4}$ nanoparticles for hyperthermia. Appl Phys Lett. 2010;96: 202511-202513.

16. Kim C, Galloway JF, Lee KH, Searson PC. Universal antibody conjugation to nanoparticles using the $\mathrm{Fc} \gamma$ receptor I (Fc $\gamma \mathrm{RI})$ : quantitative profiling of membrane biomarkers. Bioconjug Chem. 2014;25(10): 1893-1901.

17. Hwang MP, Lee JW, Lee KE, Lee KH. Think modular: a simple apoferritin-based platform for the multifaceted detection of pancreatic cancer. ACS Nano. 2013;7(9):8167-8174.

18. Jang GH, Hwang MP, Kim SY, Jang HS, Lee KH. A systematic in-vivo toxicity evaluation of nanophosphor particles via zebrafish models. Biomaterials. 2014;35(1):440-449.

19. Lee H, Sun E, Ham D, Weissleder R. Chip-NMR biosensor for detection and molecular analysis of cells. Nat Med. 2008;14(8):869-874.

20. Jun YW, Lee JH, Cheon J. Chemical design of nanoparticle probes for high-performance magnetic resonance imaging. Angew Chem Int Edit. 2008;47(28):5122-5135.

21. Laurent S, Forge D, Port M, et al. Magnetic iron oxide nanoparticles: synthesis, stabilization, vectorization, physicochemical characterizations, and biological applications. Chem Rev. 2008;108(6):2064-2110.
International Journal of Nanomedicine

\section{Publish your work in this journal}

The International Journal of Nanomedicine is an international, peerreviewed journal focusing on the application of nanotechnology in diagnostics, therapeutics, and drug delivery systems throughout the biomedical field. This journal is indexed on PubMed Central, MedLine, CAS, SciSearch $®$, Current Contents $\AA /$ Clinical Medicine,

\section{Dovepress}

Journal Citation Reports/Science Edition, EMBase, Scopus and the Elsevier Bibliographic databases. The manuscript management system is completely online and includes a very quick and fair peer-review system, which is all easy to use. Visit http://www.dovepress.com/ testimonials.php to read real quotes from published authors. 